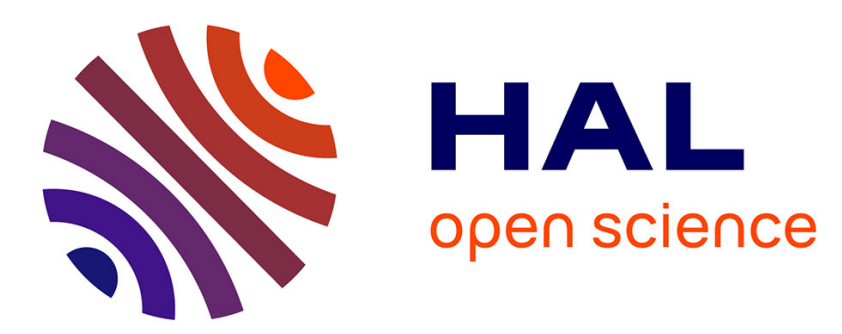

\title{
Femtosecond Photodissociation Dynamics of van der Waals Cationic Clusters: a tool for detecting metastable isomers of organic cations
}

\author{
S. Sorgues, J. M. Mestdagh, B. Soep, J. P. Visticot
}

\section{> To cite this version:}

S. Sorgues, J. M. Mestdagh, B. Soep, J. P. Visticot. Femtosecond Photodissociation Dynamics of van der Waals Cationic Clusters: a tool for detecting metastable isomers of organic cations. Chemical Physics Letters, 2004, 391, pp.254-258. 10.1016/j.cplett.2004.04.103 . hal-00084977

\section{HAL Id: hal-00084977 \\ https://hal.science/hal-00084977}

Submitted on 28 Jul 2006

HAL is a multi-disciplinary open access archive for the deposit and dissemination of scientific research documents, whether they are published or not. The documents may come from teaching and research institutions in France or abroad, or from public or private research centers.
L'archive ouverte pluridisciplinaire HAL, est destinée au dépôt et à la diffusion de documents scientifiques de niveau recherche, publiés ou non, émanant des établissements d'enseignement et de recherche français ou étrangers, des laboratoires publics ou privés. 


\title{
Femtosecond Photodissociation Dynamics of van der Waals Cationic Clusters: a tool for detecting metastable isomers of organic cations.
}

\author{
S. Sorgues, J.-M. Mestdagh, B. Soep, and J.-P. Visticot \\ Laboratoire Francis Perrin (CNRS-URA-2453), \\ DSM/DRECAM/Service des Photons, Atomes et Molécules, \\ C.E.A. Saclay, \\ F-91191 Gif-sur-Yvette cedex, France
}

\begin{abstract}
A femtosecond pump (266 nm)-probe (800 nm) experiment as been performed on small clusters of tetrakis(dimethylamino) ethylene (TDMAE) with argon (TDMAE $(\mathrm{Ar})_{\ll 10}$ ). The pump excites TDMAE and initiates deformations of the molecule within the cluster. Then, the probe ionises TDMAE and disposes enough excess energy in the cluster to evaporate all argon atoms. However, ionisation during the first $150 \mathrm{fs}$ after the pump gives preferentially access to a metastable configuration of the $\mathrm{TDMAE}^{+}$ion, unquenched by the argon environment. This takes up enough of the excess energy to prevent complete argon evaporation. Observing the non-total-evaporation of weakly bond clusters formed of an organic cation with argon appears as a useful tool to reveal the existence of such metastable configurations in organic cations.
\end{abstract}

Submitted to Chem. Phys. Lett.

Version date: April 28, 2004 


\section{Introduction}

Carbocations are strongly electrophile compounds that play an important role as reactive intermediates in organic chemistry [1]. The most important reactive site is the charge centre and documenting the existence of metastable isomers that differ by the charge location is of importance in rationalising the reactivity of excited carbocations. The present paper introduces a novel method to reveal the existence of such metastable isomers. It transposes to the femtosecond domain the technique called Photodissociation Spectroscopy of van der Waals Cationic Clusters that was proposed by Bieske et al for the spectroscopy of ions [2].

The spectroscopic technique by Bieske et al consists in forming a cold fragile cation-Ar complex. This documents the absorption spectroscopy of the cation by taking advantage of the weakness of the cation-Ar bond in two ways. First it assumes that the absorption spectroscopy of the complex closely reflects that of the free cation. Second, it assumes that the absorption of a photon by an internally converting electronic state of the cation within the complex will result inevitably in an argon atom loss that can be detected by mass spectrometry, thus offering a tool to investigate the absorption bands of the cation [2].

By analogy, we call the present technique Femtosecond Photodissociation Dynamics of van der Waals Cationic Clusters. Again it consists in forming cold fragile van der Waals clusters, but unlike the original technique, the clusters are neutral at first and are ionised later in a femtosecond pump-probe experiment. A neutral organic molecule is clustered with a few argon atoms. Then it is excited electronically by a first femtosecond laser pulse. A wavepacket is launched on the excited potential energy surface of the neutral molecule and starts moving, dragged by the potential gradients. After a certain delay the molecule is ionised by a second femtosecond laser pulse. This acts as a temporal switch that projects both the geometry of the neutral molecule and the momentum of the wavepacket into the ion space. Two dynamically different situations are expected whenever the newly formed 
ion is far or close from the bottom of a well in the energy surface of the cation. In the former case, the cation has much potential energy in excess that will be ultimately converted as heat in the cation- $\mathrm{Ar}_{\mathrm{n}}$ cluster, thus resulting into evaporation of argon atoms. By choosing small enough clusters all the argon atoms can be lost. In the second case, the cation has no excess energy above the potential well, except that due to the momentum of the wavepacket transported into the newly formed cation by the exciting photon. When this momentum is not too large, a limited evaporation is expected, even in very small clusters and a cation-Ar signal can be observed.

When performing an experiment where very small neutral clusters are generated and ionised as explained above, the observation of a cation-Ar signal as a function of the pumpprobe delay appears as a tool to document the topology of the ion. Thus it will appear that during a certain time window, a potential well of the cation can be accessed with no or little excess energy. Regarding the carbocation reactivity mentioned above, an attractive situation is encountered when this well is metastable. The present paper aims to illustrate this strategy for documenting a yet unknown metastable well in the cations of tetrakis(dimethyl amino)ethylene (named TDMAE hereafter), a molecule of structural formula

$$
\begin{aligned}
& \left(\mathrm{CH}_{3}\right)_{2} \mathrm{~N} \\
& \left(\mathrm{CH}_{3}\right)_{2} \mathrm{~N}^{\mathrm{N}}=\mathrm{C}^{\mathrm{N}\left(\mathrm{CH}_{3}\right)_{2}} \mathrm{~N}\left(\mathrm{CH}_{3}\right)_{2} .
\end{aligned}
$$

\section{$2 \quad$ Experimental}

The experimental apparatus associates a pulsed supersonic beam of TDMAE seeded in argon with a Time-Of-Flight Mass-Spectrometer (TOF-MS). It is coupled to the LUCA/SLIC femtosecond laser facility of Saclay.

The beam source conditions favour free TDMAE molecules plus a distribution of very small TDMAE $(\operatorname{Ar})_{n}$ clusters. This regime of cluster generation has been reported, for instance, by Soler et al for $\mathrm{CO}_{2}$ clusters [3]. It leads populations for the clusters of size $\mathrm{n}$ that 
decay exponentially with $\mathrm{n}$. This was checked in the present case by observing mass spectra after photo-ionisation of the cluster beam by the femtosecond lasers. The operating conditions of the beam source were such that the $\mathrm{TDMAE}^{+}$ion signal dominates, $\mathrm{TDMAE}^{+}(\mathrm{Ar})$ is measurable and $\operatorname{TDMAE}^{+}(\mathrm{Ar})_{2}$ almost not visible, whatever the ionisation conditions provided by the lasers. A 1.5 bar backing pressure of argon is used for this purpose and a $300 \mu \mathrm{m}$ nozzle. Importantly, a special care is taken on the opening time of the pulsed valve that is maintained to a minimum $(500 \mu \mathrm{s})$. Together, the lasers are fired at the beginning of the gas pulse. Of course, because of evaporation phenomena after ionisation, the neutral parents of the observed $\operatorname{TDMAE}^{+}(\mathrm{Ar})$ cluster ions are larger than TDMAE(Ar). Given the chosen clusterization regime, the cluster distribution is believed to peak at TDMAE(Ar) and its exponential tail will extend to much less than ten argon atoms per cluster.

The LUCA laser facility delivers femtosecond pulses at two colors. The pump at $266 \mathrm{~nm}$ $(4.66 \mathrm{eV})$ and the probe at $800 \mathrm{~nm}(1.55 \mathrm{eV})$ are overlapped spatially in the ionisation chamber of the TOF-MS. A delay line allows to vary the time delay between the pump and the probe pulses. The cross correlation widths of the laser pulses is $120 \mathrm{fs}$. It has been determined in the same time as the zero delay time between the pump and the probe laser using the ionisation of butadiene that is purely non resonant.

The pump laser performs a one photon excitation of the TDMAE molecule within the $\operatorname{TDMAE}(\operatorname{Ar})_{\mathrm{n}}$ clusters. The probe laser at $800 \mathrm{~nm}$ has an energy density lower than $2 \times$ $10^{11} \mathrm{~W} \mathrm{~cm}^{-2}$. It can ionise the TDMAE molecule within the TDMAE $(\mathrm{Ar})_{\mathrm{n}}$ clusters provided that TDMAE is excited electronically. The ionisation proceeds from either a one- or a twophoton ionisation process as we shall see below.

The ions stay about one microsecond in the ionisation chamber of the TOF-MS. This fixes the time scale for argon atom losses from the $\operatorname{TDMAE}^{+}(\mathrm{Ar})_{\mathrm{n}}$ cluster ions. 


\section{$3 \quad$ Results and discussion}

The $\mathrm{TDMAE}^{+}(\mathrm{Ar})$ signal is shown in Figure (1) as a function of the pump/probe delay. A monoexponential decay is observed that is fitted adequately by a single exponential of time constant $\tau_{\mathrm{A}}=150 \pm 20 \mathrm{fs}$, after convolution by the cross-correlation width of the lasers.

Before discussing the experimental results of the previous section, we must recall some of the results obtained in our laboratory on the dynamics of the TDMAE molecule either when free $[4,5]$ or coated by many argon atoms [6].

The femtosecond excitation of TDMAE at $266 \mathrm{~nm}$ promotes a $\pi$ electron of the central $\mathrm{C}=\mathrm{C}$ bond to the corresponding antibonding $\pi^{*}$ orbital. This puts the molecule in an excited potential surface, the dominant character of which is valence in the Franck Condon region of the excitation. This region is noted $\mathrm{V}\left(\pi \pi^{*}\right)$ in Figure (2). This launches a wavepacket that starts moving on the slopes of the excited potential of neutral TDMAE and the TDMAE molecule deforms along two coordinates: torsion about the CC bond and, when the wavepacket approaches a conical intersection (noted CI region in Figure (2)) with an upper surface of zwitterionic character, pyramidalization about one of the central carbon atoms. After passing the CI region, the neutral TDMAE molecule has mainly a zwitterionic character $\mathrm{C}^{+} \mathrm{C}^{-}$(noted $\mathrm{Z}$ in Figure $(2)$ ). Because of the $\mathrm{CI}$, the change of the electronic configuration, which can be viewed as a non-radiative $\mathrm{V}$ to $\mathrm{Z}$ energy transfer, occurs with no surface hopping of the wavepacket. The time constant for the $\mathrm{V}$ to $\mathrm{Z}$ energy transfer is $300 \mathrm{fs}$ [4]. Of course, the wavepacket launched on the excited surface is accelerated on the slopes of this surface. This corresponds to an increasing momentum of the neutral molecule on this coordinate, that is transported in the ion upon ionisation. This will be of importance to discuss the cluster experiment below. A last point should be made from these earlier works. With a probe laser at $800 \mathrm{~nm}$, the $\mathrm{V}$ configuration is ionised to $\mathrm{TDMAE}^{+}$in a single photon event, whereas ionisation of the $\mathrm{Z}$ configuration, which is lower in energy, needs 
two photons. This appears in Figure (2) where the adiabatic ionisation energy of TDMAE, $5.4 \mathrm{eV}$, is shown. Only the one photon ionisation of the $\mathrm{V}$ configuration is relevant here.

A previous work in our laboratory has addressed the change in the $\mathrm{V}$ to $\mathrm{Z}$ energy transfer when the TDMAE molecule is coated by many argon atoms, in an experiment where the supersonic expansion generates a log-normal distribution of TDAME $(\mathrm{Ar})_{\mathrm{n}}$ clusters broadly centered at $\mathrm{n} \approx 60[6]$. Presumably because of the weakness of the interaction between TDMAE and the argon atoms, which is essentially of van der Waals character, the argon coating does not change qualitatively the dynamics of the TDMAE molecule. Simply, by an inertial effect that we called a chistera effect in Ref. [6], it enhances the time constant of the V to Z energy transfer by $100 \mathrm{fs}$, from $300 \mathrm{fs}$ in the free molecule to $400 \mathrm{fs}$ in the molecule covered with argon.

We turn now to the present experiment where TDMAE is surrounded by less than 10 argon atoms. Likely, this limited argon environment should affect the TDMAE dynamics in a similar way, but to a lesser extent than the coating by $\approx 60$ argon atoms. Hence the time constant of the $\mathrm{V}$ to $\mathrm{Z}$ energy transfer under the present experimental conditions should be slightly larger than 300 fs. Clearly, the $150 \pm 20$ fs observed in Figure (1) does not reflect solely the $\mathrm{V}$ to $\mathrm{Z}$ energy transfer in the neutral molecule but documents an apparent lifetime of the $\mathrm{V}$ configuration, shorter than the expected one. We shall interpret this below as due to the evaporation of argon atoms that reduces the observation window of the $\mathrm{V}$ configuration when $\mathrm{TDMAE}^{+}(\mathrm{Ar})$ ions are observed. Energetics must be considered first to address this question.

Estimates of the TDMAE-Ar $\left(200 \mathrm{~cm}^{-1}=0.025 \mathrm{eV}\right)$ and TDMAE TD $^{+}$Ar $\left(450 \mathrm{~cm}^{-1}=\right.$ $0.06 \mathrm{eV}$ ) binding energies are given in Ref. [6] by comparison to binding energies measured for equivalent systems, respectively s-tetrazine- $\operatorname{Ar}\left(254 \mathrm{~cm}^{-1}[7]\right)$ and aniline ${ }^{+}-\operatorname{Ar}\left(520 \mathrm{~cm}^{-1}[8\right.$, 9]). The Ar-Ar binding energy is $98 \mathrm{~cm}^{-1}$ [10]. With these values, upper bounds of the cluster solvation energies can be found by considering the largest clusters, TDMAE $(\mathrm{Ar})_{10}$ 
and $\operatorname{TDMAE}^{+}(\mathrm{Ar})_{10}$, that are expected in the present experiment. Their solvation energy is estimated respectively to c.a. $4000 \mathrm{~cm}^{-1}(0.5 \mathrm{eV})$ and c.a. $6500 \mathrm{~cm}^{-1}(0.8 \mathrm{eV})$. Hence, the adiabatic ionisation energy of TDMAE falls from $5.4 \mathrm{eV}$ in the free TDMAE molecule [11] down to $5.1 \mathrm{eV}$ in $\operatorname{TDMAE}(\operatorname{Ar})_{10}$. The excess energy given by the one pump+one probe photons above the ionisation of $\operatorname{TDMAE}(\operatorname{Ar})_{10}$ is therefore $c a 1.1 \mathrm{eV}$. Given that much less than $0.8 \mathrm{eV}$ is needed to evaporate all the argon atoms from $\operatorname{TDMAE}^{+}(\mathrm{Ar})_{\ll 10}$ and that the cluster ions spend $1 \mu \mathrm{s}$ in the ionisation chamber of the TOF-MS, full evaporation of the argon atoms is expected when a significant fraction of the $1.1 \mathrm{eV}$ excess energy is randomized as vibrational energy in the cluster ion.

It could be thought that the $\mathrm{TDMAE}^{+}(\mathrm{Ar})$ ions observed in the present work originate from clusters present in the tail of the cluster distribution that are sufficiently large for the evaporation to be uncomplete, although all the $1.1 \mathrm{eV}$ excess energy is randomized in the cluster. The energetics above indicates that $\operatorname{TDMAE}(\mathrm{Ar})_{\gg 20}$ would be required. This is very unlikely for two reasons. First, the fraction of such clusters is close to zero under the present operating conditions. Second, when the argon backing pressure is increased and the delay between the pulsed valve and the laser is adjusted to allow such large clusters to be formed, the behaviour of $\mathrm{TDMAE}^{+}(\mathrm{Ar})$ signal reflects the full dynamics of the $\mathrm{V}$ to $\mathrm{Z}$ energy transfer (400 fs in the argon environment) [6]. Hence, the $\mathrm{TDMAE}^{+}(\mathrm{Ar})$ cluster ion signal observed in Figure (1) more likely originate from the small TDMAE $(\mathrm{Ar})_{\ll 10}$ clusters that are dominant under the present beam conditions.

Two very different possibilities may be proposed to interpret the $150 \pm 20$ fs decay of the $\operatorname{TDMAE}^{+}(\mathrm{Ar})$ signal in Figure (1). The first one is that the neutral TDMAE $(\mathrm{Ar})_{\mathrm{n}}$ parents evaporate all the $\mathrm{n}$ argon atoms after the pump pulse with a time constant of $150 \mathrm{fs}$ and can no longer lead to the $\mathrm{TDMAE}^{+}(\mathrm{Ar})$ ion upon ionisation. This is very unlikely given the weakness of the van der Waals interaction between TDMAE and the surrounding argon atoms. Indeed, the electronic excitation provided by the pump laser is localised on 
the TDMAE molecule and this energy is redistributed to the argon environment through deformation vibrations of the TDMAE molecule and quenching of the electronic energy towards lower electronic states. The time scale for this energy transfer follows the $\mathrm{V}$ to $\mathrm{Z}$ dynamics or is longer, hence more than 300 fs. The subsequent ejection of argon atoms is statistical. The time scale that is expected for the loss of all argon atoms is therefore much longer than 300 fs. This is simply not supported by the observed 150 fs time scale in the $\mathrm{TDMAE}^{+}(\mathrm{Ar})$ dynamics. Hence we favor a second possibility, in line with the strategy described in the introduction of the present work. Here the observation of non-fragmented $\mathrm{TDMAE}^{+}(\mathrm{Ar})$ cluster ions is the indication that the $\mathrm{TDMAE}^{+}$molecular ion attached to Ar was formed near the bottom of a potential well, a metastable well, with almost no momentum transferred from deformations of the neutral parent by the ionisation step.

The interpretation picture follows the three following points.

i) The pump pulse at $266 \mathrm{~nm}$ is absorbed locally by TDMAE within the TDMAE $(\mathrm{Ar})_{\ll 10}$ clusters. The excited TDMAE molecule undergoes the $\mathrm{V}$ to $\mathrm{Z}$ evolution on a time scale slightly longer than 300 fs but not sufficiently long to allow for significant argon atom losses. Then, a single $800 \mathrm{~nm}$ photon ionises TDMAE while it still keeps the V configuration. If the ionisation populates the $\mathrm{TDMAE}^{+}$component in its most stable electronic configuration, then up to $1.1 \mathrm{eV}$ excess energy can be randomized in the cluster ion as vibrational energy, as discussed earlier. Such a randomization is likely, given the structural differences between neutral TDMAE and the most stable configuration TDMAE $^{+}$ion [12]. Substantial structural differences are also expected between TDMAE ${ }^{+}$and excited TDMAE in the V configuration. Hence, vertical or nearly vertical ionisation of neutral TDMAE will form the ground state of $\mathrm{TDMAE}^{+}$ion quite far from the bottom of the corresponding potential energy surface, a situation that stimulates argon atom losses. Since the cluster of the present experiment are very small, a full evaporation of the argon atoms is expected in this case.

ii)In turn, ground state $\mathrm{TDMAE}^{+}$has the positive charge delocalised on the four nitrogen 
atoms and is noted $\operatorname{TDMAE}(-\sigma)$ in Figure (2), considering that the missing electron is taken from the lone $\mathrm{N} \sigma$ pairs. This structure has been derived from ab-initio calculations that have also predicted the existence of a secondary minimum on the same potential energy surface with the charge delocalisation on two diametrically opposite nitrogen atoms [12]. An electronically excited configuration of the $\mathrm{TDMAE}^{+}$ion may be imagined also when considering that vertical ionisation from the $\mathrm{V}$ electronic configuration of TDMAE would certainly eject the $\pi^{*}$ electron and form the

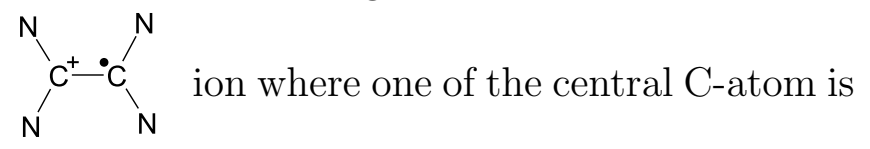
positive. This configuration is noted $\operatorname{TDMAE}(-\pi)$ in Figure (2) is likely excited with respect to the TDMAE $(-\sigma)$ configuration. This is justified given that the ionisation of alkenes mimics the $\operatorname{TDMAE}(-\pi)$ configuration and occurs at larger energies than the adiabatic ionisation energy of TDMAE, $8.4 \mathrm{eV}$ for tetramethylethylene $\left(\mathrm{CH}_{3}\right)_{2} \mathrm{C}=\mathrm{C}\left(\mathrm{CH}_{3}\right)_{2}[13]$ versus $5.4 \mathrm{eV}$ for TDMAE [11]. The situation with TDMAE would then be akin to that encountered in the pyridine ion where the lowest electronic states correspond to different electron holes in either the nitrogen non bonding orbital or the delocalized CC bonding orbitals [14, 15].

iii)From the above considerations, we infer that a fraction of the ionisation events at short time delays between the pump and the probe laser, gives access to an excited configuration of the $\mathrm{TDMAE}^{+}$ion, possibly $\operatorname{TDMAE}(-\pi)$. Apparently ions formed in this way have a low enough vibrational energy and (or) the energy barrier preventing their internal conversion to the ground state is large enough to prevent this conversion in the presence of argon. The consequence is that a substantial fraction of the available $1.1 \mathrm{eV}$ above ionisation is stored in the ion and is not transferred to the argon atoms, thus allowing for $\mathrm{TDMAE}^{+}(\mathrm{Ar})$ to be be observed as a minor channel. When the ionisation is performed beyond $150 \mathrm{fs}$ after the pump pulse, the excited TDMAE is still in the $\mathrm{V}$ configuration and the same $\operatorname{TDMAE}(-\pi)$ configuration of the ion is still accessed. However momentum has been gained by the wavepacket descending along the excited potential energy surface of neutral TDMAE 
molecule. It is transported in the $\mathrm{TDMAE}^{+}$ion by ionisation. Apparently, after 150 fs this momentum is large enough for the small energy barrier mentioned above to be overcome. Then, full evaporation of the $\mathrm{TDMAE}^{+}(\mathrm{Ar})_{\mathrm{n}}$ clusters proceeds and no $\mathrm{TDMAE}^{+} \mathrm{Ar}$ ions are detected anymore.

\section{Summary and conclusion}

The supersonic expansion of a mixture of tetrakis(dimethylamino)ethylene (TDMAE) with argon has been used to generate small TDMAE $(\mathrm{Ar})_{\mathrm{n}}$ clusters $(\mathrm{n} \ll 10)$. The TDMAE molecule is excited and ionised within the argon cluster in a femtosecond pump (266 nm)probe $(800 \mathrm{~nm})$ experiment. When the ionisation forms the $\mathrm{TDMAE}^{+}$ion, far from the equilibrium geometry of its most stable configuration, enough potential energy is deposited into the $\mathrm{TDMAE}^{+}(\mathrm{Ar})_{\ll 10}$ cluster and transformed as vibrational excitation for a full argon evaporation to occur. However, during the first 150 fs of the excited state dynamics of TDMAE, a metastable configuration of the $\mathrm{TDMAE}^{+}$ion can be accessed, which is not quenched by the argon environment. Enough energy stays stored by the metastable configuration to prevent the full evaporation of the argon atoms and $\mathrm{TDMAE}^{+}(\mathrm{Ar})$ could be observed. This has offered a tool to monitor the existence of the metastable configuration. This can be seen as an interesting extension of Bieske's method or trapping of intermediates by matrix isolation.

We think that observing the non-total-fragmentation of such van der Waals clusters formed of an organic cation bonded to a few argon atoms could be useful to reveal the existence of metastable configurations in organic cations as presently exemplified. It could be thought that observing photoelectron spectra in a femtosecond pump-probe experiment performed on the cluster free molecule is an even more sensitive way of documenting these configurations. This is partly true only, given that electron spectra document the energy

difference between the ion energy surface and the energy surface of the neutral molecule 
where the wavepacket is launched by the pump laser. When the latter surface is hilly, the interpretation of the photoelectron spectra becomes very tricky. The present method is then very attractive since it documents the ion surface specifically.

\section{Acknowledgments}

The authors thank O. Gobert, P. Meynadier and M. Perdrix, who are in charge of the femtosecond laser facility LUCA (Laser Ultra-Court Accordable) of the CEA, DSM/DRECAM/SPAM. Partial support by the European Community is acknowledged through the PICNIC network

(Product Imaging and Correlation: Non-adiabatic Interactions in Chemistry ) under contract number HPRN-CT-2002-00183.

\section{References}

[1] N. S. Isaacs, Physical Organic Chemistry, Longman Scientific and Technical, Essex CM20 2JE, UK, 1995.

[2] E. J. Bieske, M. W. Rainbird, and A. E. W. Knight, J. Phys. Chem. 94, 3962 (1990).

[3] M. Soler, N. García, O. Echt, K. Sattler, and E. Recknagel, Phys. Rev. Lett. 49, 1857 (1982).

[4] B. Soep, J. M. Mestdagh, S. Sorgues, and J. P. Visticot, Eur. Phys. J. D 14, 191 (2001).

[5] S. Sorgues, J. M. Mestdagh, J. P. Visticot, and B. Soep, Phys. Rev. Lett. 91, 3001 (2003).

[6] S. Sorgues, J. M. Mestdagh, B. Soep, and J. P. Visticot, Submitted to Chem. Phys., 2003.

[7] D.V. Brumbaugh, J.E. Kenny, and D.H. Levy, J. Chem. Phys. 78, 3415 (1983).

[8] S. Douin, P. Parneix, and P. Bréchignac, Z. Phys. D 21, 343 (1991). 
[9] N. Solca and O. Dopfer, Eur. Phys. J. D 20, 469 (2002).

[10] U. Buck, Elastic scattering, in Molecular Scattering; Physical and Chemical Applications, edited by K. P. Lawley, chapter , pages 313-388, Wiley, Chichester,Sussex,England, 1975.

[11] Y. Nakato, M. Ozaki, and H. Tsubomura, Bull. Chem. Soc. Jap. 45, 1229 (1972).

[12] K. I. Pokhodnia, J. Papavassiliou, P. Umek, A. Omerzu, and D. Mihailovic, J. Chem. Phys. 110, 3606 (1999).

[13] M. B. Robin, Higher Excited States of Polyatomic Molecules, Vol. 2, Academic press, New York, USA, 1975.

[14] M. I. Al-Joboury and D. W. Turner, J. Chem. Soc. 4, 4434 (1964).

[15] I. C. Walker, M. H. Palmer, and A. Hopkirk, Chem. Phys. 141, 365 (1989). 


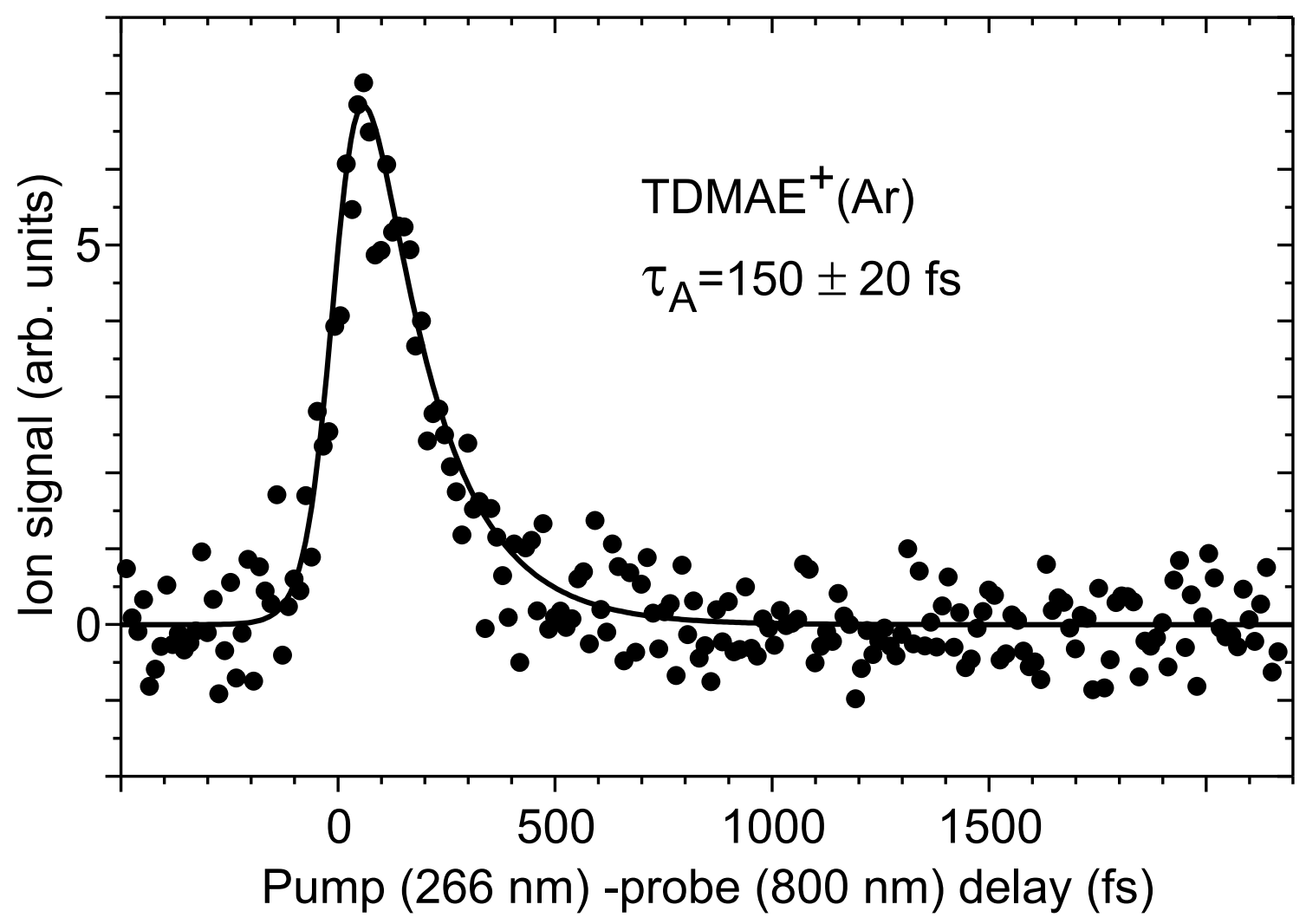

Figure 1: Evolution of the signal measured at the mass of $\operatorname{TDMAE}^{+}(\mathrm{Ar})$ as a function of the pump (266 nm)-probe (800 nm) delay. The full curve passing through the experimental points is a fit by a single exponential of decay time constant $\tau_{\mathrm{A}}=150 \pm 20 \mathrm{fs}$ convoluted by the cross correlation width of the lasers. 


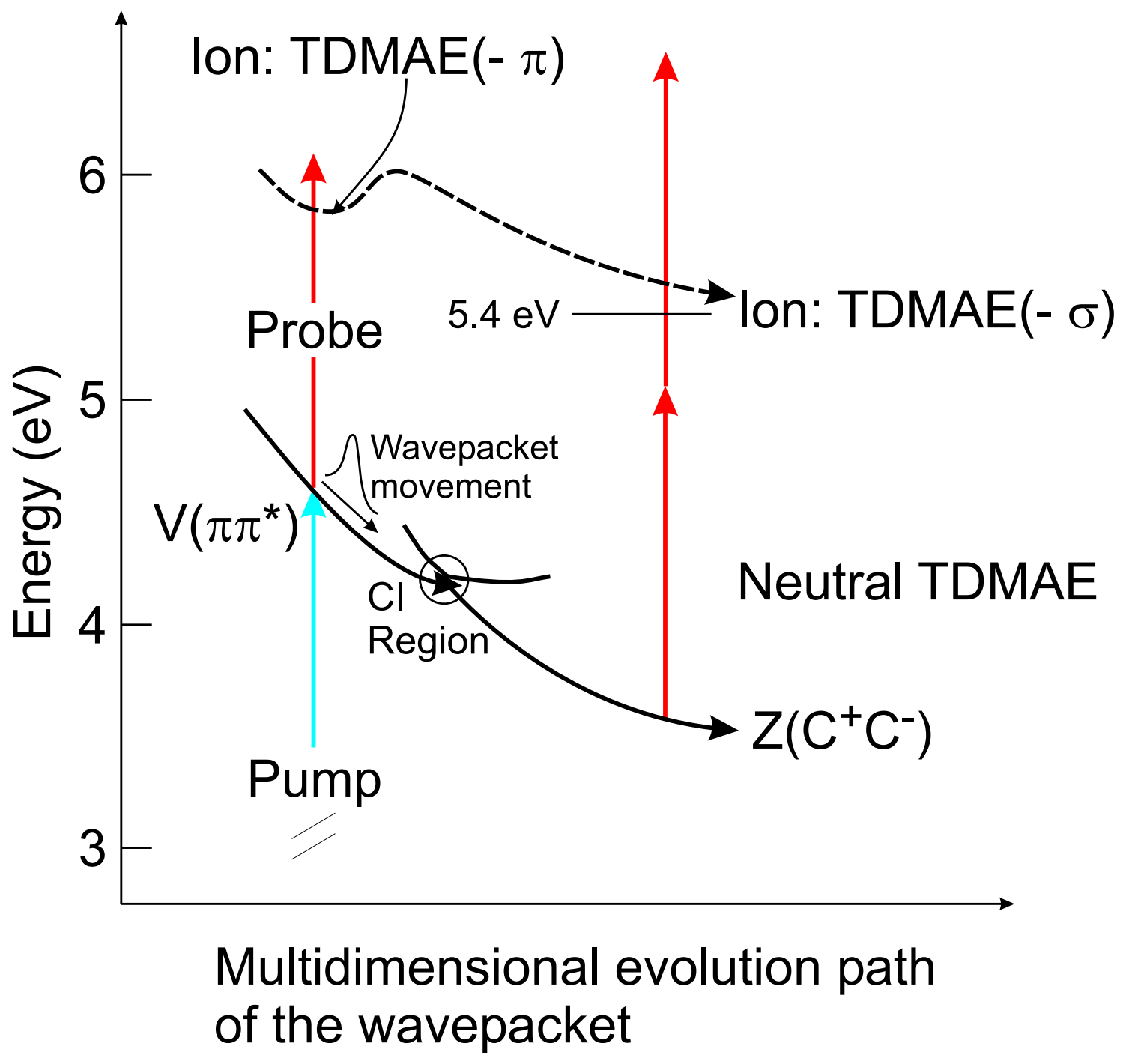

Figure 2: Scheme illustrating the dynamics of free TDMAE (see the discussion for details). 